\title{
IgM antibody level against proinflammatory bacterial peptidoglycan is inversely correlated with extent of atherosclerotic disease
}

\author{
Manon M. Oude Nijhuis ${ }^{\mathrm{a}, b, d}$, Yolanda van der Graaf ${ }^{\mathrm{c}}$, Marie-José Melief ${ }^{\mathrm{d}}$, \\ Arjan H. Schoneveld ${ }^{\mathrm{a}, \mathrm{b}}$, Dominique P.V. de Kleijn ${ }^{\mathrm{a}, \mathrm{b}}$, Jon D. Laman ${ }^{\mathrm{d}}$, Gerard Pasterkamp ${ }^{\mathrm{a}, *}$ \\ for the SMART Studygroup, members listed in ${ }^{1}$ \\ ${ }^{a}$ Experimental Cardiology Laboratory, Heart Lung Center Utrecht, University Medical Center Utrecht, Room G02.523, \\ Heidelberglaan 100, Utrecht 3584 CX, The Netherlands \\ ${ }^{\mathrm{b}}$ Interuniversity Cardiology Institute of the Netherlands, Utrecht, The Netherlands \\ c Julius Center, University Medical Center Utrecht, The Netherlands \\ ${ }^{\mathrm{d}}$ Department of Immunology, Erasmus MC, Rotterdam, The Netherlands
}

Received 24 June 2003; accepted 9 December 2003

\begin{abstract}
Objective: Atherosclerosis may lead to acute clinical events by rupture of a vulnerable atherosclerotic plaque. Previously, we demonstrated that peptidoglycan (PGN), a major cell wall component of gram-positive bacteria that induces production of proinflammatory cytokines through TLR2 and CD14, is prevalent in atherosclerotic lesions with histological features associated with plaque vulnerability. We hypothesized that in atherosclerotic patients antibody levels against PGN may differ compared with matched controls. Methods and results: ELISA was performed to measure immunoglobulin levels against PGN in sera of 80 atherosclerotic patients versus 77 control patients with an increased cardiovascular risk, frequency-matched for age, sex and risk factors for atherosclerotic disease. In all patients and controls, intima-media (IMT) thickness was assessed using an array transducer. Significantly lower levels of IgM directed against PGN were found in atherosclerotic patients compared with the control patients without clinically manifested disease $(P=0.02)$. The IgM levels against PGN decreased with increasing mean common carotid IMT thickness $(P=0.006)$. Conclusions: These results show that patients suffering from atherosclerotic disease have decreased IgM levels against PGN. The data suggest that an antibody response against PGN could have a protective effect against the development or activity of atherosclerotic disease.
\end{abstract}

(C) 2004 Elsevier Ireland Ltd. All rights reserved.

Keywords: Atherosclerosis; Gram-positive bacteria; Inflammation; Risk factors; Immune system

\section{Introduction}

Atherosclerosis is a chronic inflammatory disease [1]. During the inflammatory response mediators such as chemokines, cytokines and growth factors are released.
These mediators are able to recruit inflammatory cells such as T-lymphocytes and macrophages to the site of injury [2].

Peptidoglycan (PGN) may promote chronic inflammation at non-mucosal sites [3]. This antigen is present in the cell walls of most bacteria and is the major constituent (30-70\%)

Abbreviations: PGN, peptidoglycan; TLR, toll-like receptor; SMART, Second Manifestations of ARTerial disease; IMT, intima-media thickness; Ig, immunoglobulin; ABPI, ankle-brachial pressure index

* Corresponding author. Tel.: +31-30-250-7155; fax: +31-30-252-2693.

E-mail address: g.pasterkamp@hli.azu.nl (G. Pasterkamp).

${ }^{1}$ A. Algra (M.D., Ph.D.), Julius Center for General Practice and Patient Oriented Research and Department of Neurology, University Medical Center Utrecht, Utrecht, The Netherlands; J.D. Banga (M.D., Ph.D.), F.L.J. Visseren, T.J. Rabelink, Department of Internal Medicine, University Medical Center Utrecht, Utrecht, The Netherlands; B.C. Eikelboom (M.D., Ph.D.), Department of Vascular Surgery, University Medical Center Utrecht, Utrecht, The Netherlands; Y. van der Graaf (M.D., Ph.D.), D.E. Grobbee (M.D., Ph.D.), Julius Center for General Practice and Patient Oriented Research, University Medical Center Utrecht, Utrecht, The Netherlands; P.P.Th. de Jaegere (M.D., Ph.D.), Department of Cardiology, University Medical Center Utrecht, Utrecht, The Netherlands; L.J. Kappelle (M.D., Ph.D.), Department of Neurology, University Medical Center Utrecht, Utrecht, The Netherlands; H.A. Koomans (M.D., Ph.D.), Department of Nephrology, University Medical Center Utrecht, Utrecht, The Netherlands. 
of gram-positive cell walls. PGN surrounds the cell and gives strength to withstand the turgor pressure exerted by the cytoplasm. During gram-positive infections, PGN can activate granulocytes [4] and upregulate the expression of adhesion molecules on endothelial cells [5]. PGN can induce polyclonal antibody formation in peripheral blood mononuclear cells of healthy donors dependent on the presence of T-lymphocytes and monocytes [6]. Furthermore, PGN is able to induce production of pro-inflammatory cytokines such as IL-1, IL- 6 and TNF- $\alpha$ by monocytes/macrophages in vitro through engagement of toll-like receptor 2 (TLR2) and CD14 [7,8]. These macrophages are crucial in lipid metabolism and the development of atherosclerotic lesions [9]. Recently, we demonstrated that PGN is present in atherosclerotic plaques with histological features of a vulnerable plaque phenotype [10]. Furthermore, PGN may play a role in the pathogenesis of rheumatoid arthritis, which also is a chronic inflammatory disease [11,12]. In view of the postulated functional roles of antibodies against self antigens in atherosclerosis, we hypothesized that levels of antibodies directed against PGN may be different in atherosclerotic patients as compared to matched controls. In the present study, we determined systemic antibody levels against PGN in patients with severe atherosclerotic disease and compared these levels to those in a control group without clinically manifest disease. Furthermore, we examined a possible relation between the immune response against PGN and the extent of atherosclerotic disease using duplex wall thickness measurements of the carotid artery.

\section{Methods}

\subsection{Patients}

All patients were participants of the Second Manifestations of ARTerial disease (SMART) study; an ongoing, single-center, prospective cohort study of approximately 3500 patients referred to the University Medical Center Utrecht for the first time because of atherosclerotic vascular disease or treatment of atherosclerotic risk factors [13]. The study was approved by the ethics committee of the University Medical Center Utrecht, and written informed consent was obtained from all participants.

Eighty atherosclerotic patients that fulfilled all of the following three criteria were selected: (1) a history of clinically manifest atherosclerotic disease; (2) atherosclerotic disease at the time of hospital-entry and (3) one cardiovascular risk factor (hypertension, diabetes mellitus or hyperlipidemia). Patients were additionally screened for the presence of clinically silent atherosclerotic lesions in the carotid and femoral arteries and aorta using three approaches. First, atherosclerotic luminal narrowing in the femoral artery was evaluated by measuring the ratio of the systolic blood pressure measured at the ankle to the systolic blood pressure measured in both arms (ankle-brachial pressure index, ABPI). When pa- tients had a lowered ABPI $(\leq 0.9)$ they were classified as having silent atherosclerosis. Second, stenosis of the common and internal carotid arteries was measured bilaterally with Doppler-assisted duplex scanning. When carotid stenosis exceeded $70 \%$, patients were diagnosed having clinically silent atherosclerosis. Third, ultrasonography of the abdomen was performed to measure the anteroposterior juxtarenal diameter and the distal anteroposterior diameter of the aorta. When distal anteroposterior diameter $\geq 3 \mathrm{~cm}$ or $\geq 1.5$ times anteroposterior juxtarenal diameter, patients were diagnosed having clinically silent atherosclerosis.

Seventy-seven control patients from the SMART study were frequency-matched for sex, age and one of three major risk factors (hypertension, diabetes mellitus and hyperlipidemia). These patients revealed neither clinically evident nor silent atherosclerosis.

\subsection{Isolation of PGN from human intestinal flora}

PGN was prepared from faeces of a healthy subject [14]. In short, homogenized faeces was filtrated and centrifuged for $45 \mathrm{~min}$ at $5000 \times \mathrm{g}$. Four volumes of $96 \%$ ethanol were added and after $2 \mathrm{~h}$ the precipitate was centrifuged for $15 \mathrm{~min}$ at $5000 \times g$. After dissolving in Milli-Q the suspension was centrifuged for $1 \mathrm{~h}$ at $100,000 \times g$. Size exclusion chromatography was done on the clear supernatant using dilutions of $15-60 \mathrm{mg} / 30 \mathrm{ml}$. Collected fractions containing carbohydrates but no proteins were pooled, dialyzed and lyophilized. About $50 \mathrm{mg}$ PGN could be retrieved from $100 \mathrm{~g}$ of faeces. This represents a full representation of the multitude of bacterial species occurring in the normal intestinal flora. Since PGN structure varies from one bacterial species to another, PGN derived from the full flora provides a broad range of PGN structures.

\subsection{Enzyme-linked immunosorbent-assay (ELISA) for anti-PGN level}

The direct ELISA with faeces PGN coated to microtiter wells to measure anti-PGN level has been developed and validated previously $[11,15]$. As a reference for calculating antibody levels against PGN, a pooled standard serum $(\approx 500$ bloodbank donors) selected for high anti-PGN reactivity was used. In microtiter plates $50 \mu \mathrm{l}, 10 \mu \mathrm{g} / \mathrm{ml}$ PGN diluted in PBS was coated overnight at $50^{\circ} \mathrm{C}$. One hundred microliter serum diluted in PBS/Tween $0.2 \%$ was added and incubated for $1 \mathrm{~h}$ at $37^{\circ} \mathrm{C}$. Dilutions of 1:400, 1:1600 and 1:200 were used for IgM, IgG and IgA measurements, respectively. Peroxidase conjugated rabbit anti-human IgM, IgG or IgA (Jackson Immunoresearch, Inc., Westgroove, PA) diluted in PBS/Tween $0.2 \%$ was used as secondary antibody during $1 \mathrm{~h}$ at $37^{\circ} \mathrm{C}$. The development of the colorimetric assay took place in the dark for 10-20 min after the addition of $100 \mu \mathrm{l}$ of substrate with ortho-phenylene diamine $/ \mathrm{H}_{2} \mathrm{O}_{2}$. The reaction was stopped by adding $50 \mu 14 \mathrm{M} \mathrm{H}_{2} \mathrm{SO}_{4}$. Optical density was measured at $492 \mathrm{~nm}$. On each plate the stan- 
dard serum was included and the background absorption of the conjugate was tested in duplicate. Levels of antibodies were expressed as a ratio calculated as follows:

ratio $=\frac{\mathrm{OD}_{\text {sample }}-\mathrm{OD}_{\text {background }}}{\mathrm{OD}_{\text {standard serum }}-\mathrm{OD}_{\text {background }}}$

\subsection{Total immunoglobulin determination}

To examine whether total immunoglobulin concentrations were in the normal range, total $\operatorname{IgM}$ and $\operatorname{IgG}$ concentrations of all patients were measured using immunoephelometry according to routine and validated clinical chemistry protocols.

\subsection{Risk score}

Previously, a risk score based on data of pre-existing disease and risk factors available in the SMART cohort (SMART risk score) was developed and validated by comparing the SMART risk score to the Framingham risk score and the EPOZ risk score [16]. The three risk scores strongly correlated with each other. Single points were given for male gender, age, cardiovascular risk factors, and history and presence of cardiovascular disease, to a maximum of 24 points (Table 1). If more than two risk indicators were missing, the risk score was classified as missing ( $n=9$ patients). If two or fewer risk indicators were missing, the missing indicator was given zero points ( $n=1$ patient).

\subsection{Intima-media thickness measurement}

In all patients intima-media thickness (IMT) was measured in the left and right common carotid arteries as a measure for the extent of atherosclerosis. Arteries were examined in anterolateral, posterolateral and mediolateral direction using an ATL Ultramark 9 machine (Advanced Technology Laboratories, Bethel, WA) equipped with a $10 \mathrm{MHz}$ linear array transducer. The intima-media surface of the selected area was calculated on-line using built-in software of the ultrasound system. The mean IMT of the six measurements in each patient was calculated. Investigators who performed the ELISAs were blinded for the outcome of IMT measurements.

\subsection{Statistical analysis}

A Mann-Whitney test was used to compare the ratios of IgM, IgG and IgA measured in the PGN-ELISA of the atherosclerotic patients and the control group versus standard serum. All patients were divided into quartiles on the basis of IMT or SMART risk score. Differences in IgM levels against PGN between the quartiles were measured with one-way ANOVA and a Tukey post hoc multiple comparison. All values are presented as mean \pm standard error of mean (S.E.M.). A $P$-value of $<0.05$ was considered significant.
Table 1

SMART risk score based on pre-existing vascular disease and risk factors

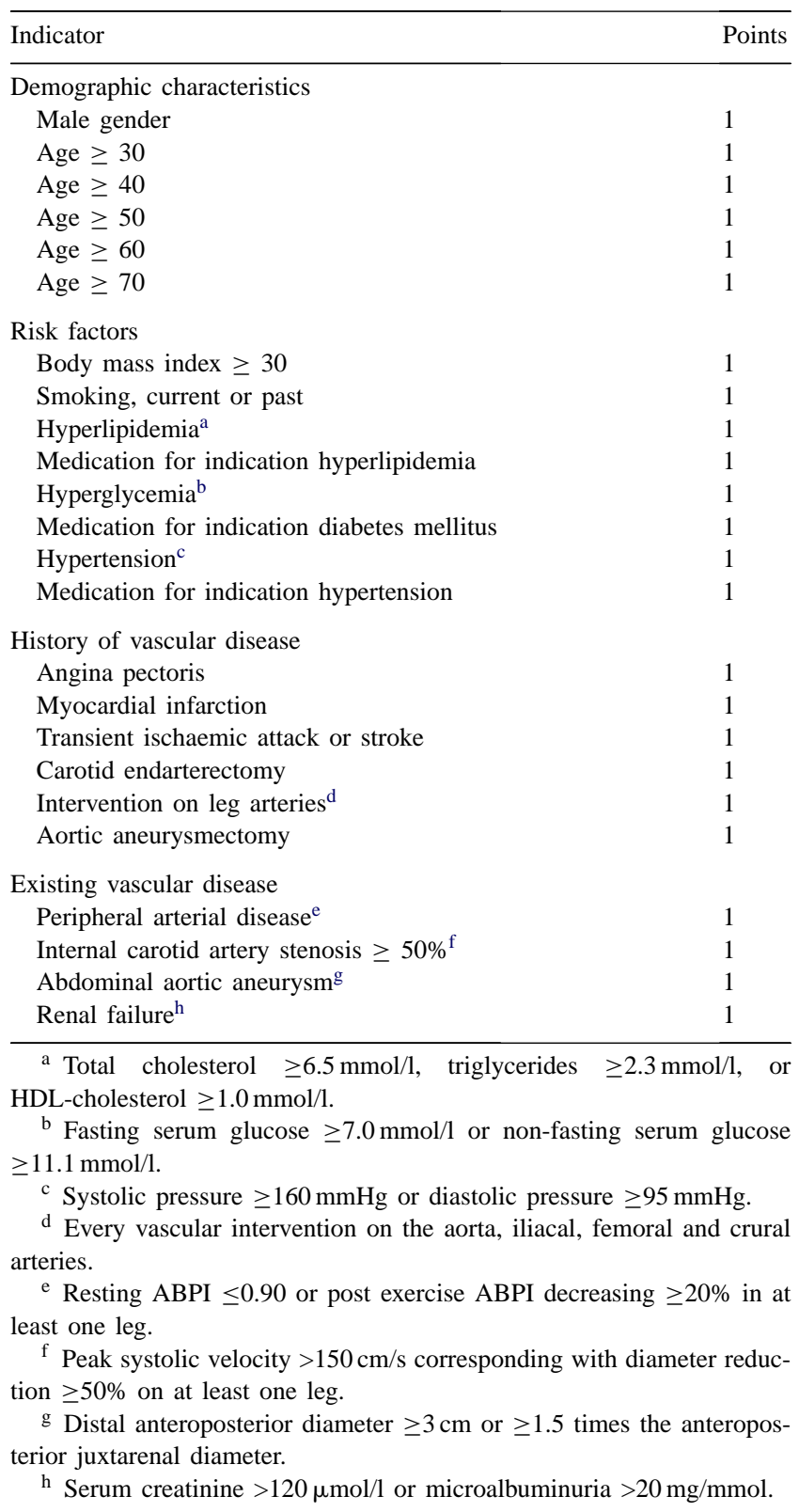

\section{Results}

\subsection{Systemic antibody level against PGN in atherosclerotic patients}

The study population consisted of 80 atherosclerotic patients (40 males, 40 females; mean age 60, range 31-79) and 77 sex-, age-, and risk- factor-matched control patients without clinically manifest disease ( 38 males, 39 females; mean age 58, range 32-76). Forty, thirty-one and six atherosclerotic patients entered the hospital with asymptomatic carotid stenosis, peripheral arterial disease and abdominal aortic aneurysm, respectively. Furthermore, 5, 7 and 68 atherosclerotic patients suffered from hypertension, diabetes and hy- 
Table 2

Characteristics of the study population

\begin{tabular}{lll}
\hline Characteristics of the study population & Controls & Cases \\
\hline Total number & 77 & 80 \\
Age (years) & 57.5 & 59.7 \\
Women (\%) & 49 & 50 \\
Body mass index $\left(\mathrm{kg} / \mathrm{m}^{2}\right)$ & 26.1 & 26.1 \\
Smoking $(\%)$ & 29 & 50 \\
Mean common carotid intima-media & 0.078 & 0.104 \\
$\quad$ thickness (cm) & & 9.96 \\
SMART risk score & 6.77 & \\
\hline
\end{tabular}

perlipidemia, respectively. In Table 2, the baseline characteristics of the study population are presented. The data indicate that atherosclerotic patients have significantly lower IgM antibody levels against PGN compared to control patients $(0.527 \pm 0.037$ versus $0.630 \pm 0.039 P=0.02)$. No differences were observed in IgG and IgA antibody levels against PGN in atherosclerotic patients compared to control patients $(0.400 \pm 0.023$ versus $0.348 \pm 0.023 P=0.1$ for $\mathrm{IgG}$ and $0.853 \pm 0.074$ versus $0.932 \pm 0.079 P=0.4$ for IgA, respectively). Total IgM and IgG immunoglobulin concentrations of the control patients were not significantly different from atherosclerotic patients $(1.26 \pm 0.07 \mathrm{~g} / 1$ versus $1.09 \pm 0.07 \mathrm{~g} / 1, P=0.1$ and $9.93 \pm 0.34 \mathrm{~g} / 1$ versus $10.13 \pm 0.28 \mathrm{~g} / \mathrm{l}, P=0.6$, respectively). Furthermore, no relation between $\operatorname{IgM}, \operatorname{IgG}$ and $\operatorname{IgA}$ antibody levels against PGN and age was observed $(P=0.07,0.9$ and 0.2 , respectively). In the same patient cohort at another timepoint, measurement of IgM levels against PGN could be reproduced $(0.711 \pm 0.055$ versus $0.860 \pm 0.057$, atherosclerotic patients versus control patients $P=0.03$ ). For repeat experiments both primary optical density readings as well as ratio of patient over reference signals were ranked for all patients. No differences between the experiments were observed in the rankings, demonstrating that the experiments were comparable and the data reproducible.

\subsection{Antibody titers against $P G N$ in relation to IMT and SMART risk score}

Fig. 1 presents the association between common carotid IMT and IgM antibody level against PGN in all patients. The cut-off values of the IMT run from 0.05 to $0.06 \mathrm{~cm}$ in quartile 1 , from 0.07 to $0.09 \mathrm{~cm}$ in quartile 2 , from 0.10 to $0.12 \mathrm{~cm}$ in quartile 3 and from 0.13 to $0.15 \mathrm{~cm}$ in quartile 4. The IgM level against PGN decreased with increasing mean IMT $(P=0.006)$; the $P$ for trend is 0.008 .

The decrease was significant between the first and third and between the first and fourth quartile $(P=0.02$ and 0.004 , respectively). After normalization of the specific IgM response against PGN to the total IgM response, a decrease could still be observed with increasing IMT $(P=0.009)$. At the second time point these data could be reproduced. The IgM level against PGN decreased with increasing mean IMT $(P=0.001)$. Between the first and third and between the

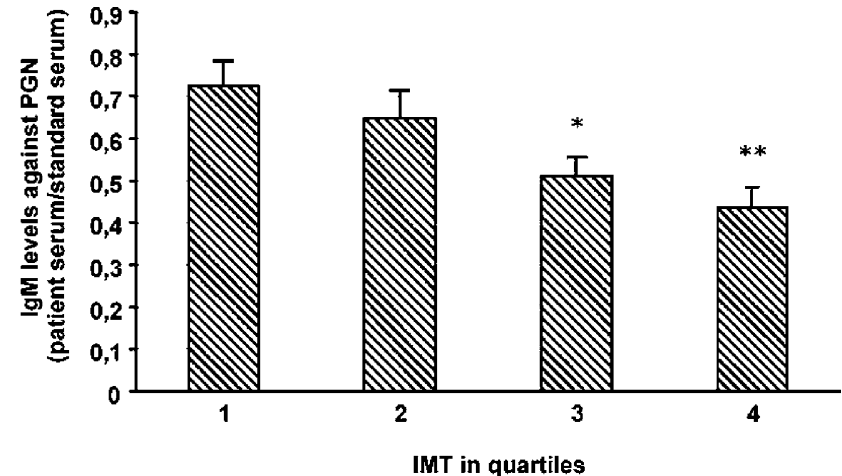

Fig. 1. IgM level against peptidoglycan (PGN) in relation to common carotid intima-media thickness (IMT). The IgM level against PGN decreased with increasing IMT $\left(R^{2}=0.0537, P=0.006, P\right.$ for trend is $0.008)$. The decrease was significant between the first and third and first and fourth quartile $\left({ }^{*} P=0.02\right.$ and $\left.{ }^{* *} P=0.004\right)$.

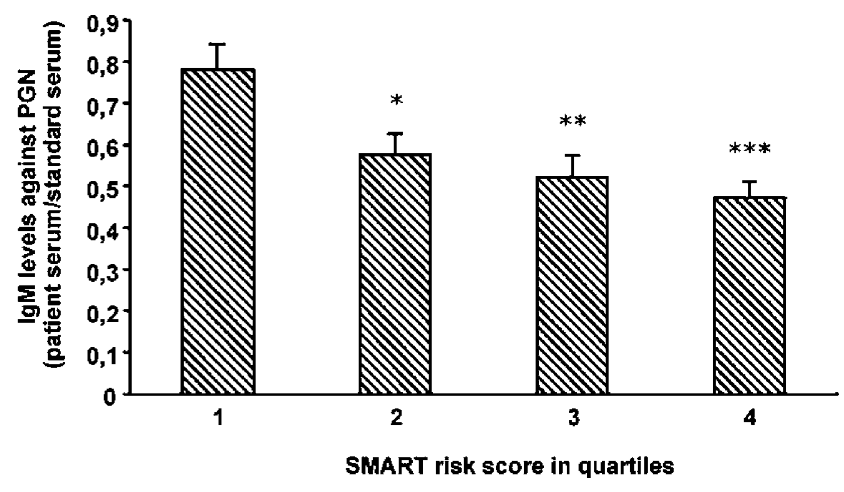

Fig. 2. IgM level against PGN in relation to SMART risk score. The SMART risk score showed an inverse relation with $\operatorname{IgM}$ levels against PGN $\left(R^{2}=0.0834, P=0.003, P\right.$ for trend is $\left.<0.001\right)$. Differences were significant between the first and second, first and third and first and fourth quartiles $\left({ }^{*} P=0.003,{ }^{* *} P=0.003\right.$ and $\left.{ }^{* * *} P<0.001\right)$.

first and fourth quartile, this decrease was again significant ( $P=0.01$ and 0.009 , respectively).

Fig. 2 demonstrates the relationship between IgM levels against PGN and the SMART risk score in all patients. The IgM level against PGN decreased with increasing SMART risk score $(P=0.003)$; the $P$ for trend is $<0.001$. The significant decrease in IgM levels against PGN was due to significant differences between the first and second, first and third and between the first and fourth quartiles $(P=0.003$, 0.003 and $\mathrm{P}<0.001$, respectively). Again after normalization of the specific IgM response against PGN to the total IgM response, the IgM response decreased with increasing SMART risk score $(P=0.02)$.

\section{Discussion}

Previously, we have demonstrated that the bacterial cell wall component PGN is present in atherosclerotic plaques [10]. Since individual systemic immunoglobulin isotypes are involved in the process of immune exclusion, i.e. prevention 
of redistribution of microbial antigens from the mucosa to other sites in the body [17], we hypothesized that atherosclerotic patients may differ from matched controls in their levels of antibodies directed against PGN. We indeed observed that atherosclerotic patients had a significantly lower systemic IgM antibody level against PGN than control patients closely matched for risk factors, but comparable IgG and IgA. In addition, the level of IgM antibodies against PGN negatively correlated with common carotid IMT and a risk score for atherosclerotic disease, suggesting a functional relationship. The results show that there is no statistically significant difference in total IgM concentration. There was however a trend towards a decrease in total $\operatorname{IgM}$ in atherosclerotic patients as well as a trend toward a decrease in IgM levels against PGN with age. Since these trends might partly explain the lowered IgM PGN titers we also normalized the specific IgM to the total IgM. After normalization a decrease in IgM levels could still be observed with increasing common carotid IMT and SMART risk score.

There are three obvious interpretations of these findings: first, the decrease in antibody levels to PGN in atherosclerotic patients might be due to a generalized depression of antibody levels. However, as mentioned earlier, even after correction for total IgM the difference in IgM levels against PGN among groups was still evident. Second, decreased IgM antibody levels against PGN might be explained by decreased exposure to the antigen. This possibility is hard to address, but seems unlikely with respect to the ubiquitous nature of PGN, being present at high level in all gram-positives and low level in gram-negatives to which we are continuously exposed at all mucosa, including the healthy intestinal and skin flora. The unaltered levels of $\mathrm{IgG}$ and $\mathrm{IgA}$ specific for PGN also argue against this option. And third, the decreased IgM levels may reflect an increased consumption of anti-PGN IgM antibodies, caused by binding to intact bacteria or cell wall fragments with subsequent immune complex formation and enhanced removal.

To our knowledge, this is the first study that has examined the relationship between the presence of immunoglobulins against PGN and atherosclerosis. Other studies also demonstrated significantly lower titers of $\operatorname{IgM}$ against oxidized LDL (Ox-LDL), a key autoantigen with neo-epitopes, in patients with a history of atherosclerosis, as well as negative associations between levels of IgM Ox-LDL antibodies and IMT $[18,19]$. Another study indicated that low IgM and IgG antibody levels against Ox-LDL may be associated with the presence of major risk factors for developing atherosclerotic disease [20]. These results support the concept that a lowered antibody response to proinflammatory antigens like PGN or Ox-LDL may be causally related to the activity of atherosclerotic disease.

In a few other studies, immunization with Ox-LDL was found to be correlated with inhibition of the progression of atherosclerosis. In mice and rabbits, it has been demonstrated that immunization with Ox-LDL leads to increased immunoglobulin levels and immunization with both
Ox-LDL and HSP-65 inhibits progression of atherosclerosis [21,22]. Furthermore, Caligiuri et al. [23] suggest that protection from atherosclerosis is associated with specific antibody responses to Ox-LDL, developing in the spleen. This is further supported by Witztum and coworkers [24] who reported that $\operatorname{IgM}$ antibodies reactive to phosphorylcholine are deposited in atherosclerotic lesions, can recognize Ox-LDL and block its uptake by macrophages. These results suggest that an immune response against (auto) antigens has a protective effect against the development of atherosclerotic lesions. In this study, we showed the opposite, a lowered immune response to the antigen PGN is associated with more pronounced atherosclerotic disease. While Ox-LDL and HSP-65 are auto-antigens, PGN is the first exogenous antigen that shows a negative correlation between specific IgM and atherosclerotic activity. However, the role of the immune system in the pathogenesis of atherosclerosis is complex [25]. Therefore, we can not conclude that all increased antibody responses are protective, while decreased antibody responses are pro-atherogenic.

In atherogenesis, the earliest changes are observed in the subendothelial matrix where fatty streaks develop with the invasion of monocytes, T-lymphocytes and smooth muscle cells. The fatty streak progresses into an advanced lesion with necrotic core formation that is covered by a fibrous cap. Eventually a plaque can rupture, leading to unstable coronary syndromes or myocardial infarction (MI). Previously, we studied PGN expression in advanced lesions [10] and demonstrated that PGN is present in lesions with an inflammatory, vulnerable, plaque phenotype. In this study, we found that in atherosclerotic patients the $\operatorname{IgM}$ antibody levels against PGN are significantly lower compared to the control patients, suggesting that the presence of PGN is associated with a decreased IgM antibody titer against PGN. It is unknown whether the results of the present study can be applied not only to advanced lesion formation but also to the early stages of atherosclerotic disease. It is highly likely that any control population will also hide early stage atherosclerotic lesions so this answer will probably be given by post mortem studies in which non-atherosclerotic segments need to be investigated for the presence of PGN.

Other research groups showed previously that PGN may play a role in the pathogenesis of rheumatoid arthritis, which also is a chronic inflammatory disease $[11,12]$. In these studies rheumatoid arthritis patients had significantly lower systemic IgG antibody levels against PGN compared to healthy controls.

Previous studies suggest that an association exists between chronic infection with Chlamydia pneumoniae and manifestations of coronary heart disease (CHD) [26,27]. $C$. pneumoniae has been found within atherosclerotic tissues and high-titer IgG antibodies against $C$. pneumoniae are associated with an increased risk of CHD death and MI [28]. Furthermore, few studies have suggested there is a strong correlation between Helicobacter pylori infection and adverse cardiovascular events or systemic inflammation mark- 
ers [29,30]. Most attention has been paid to the potential role of gram-negative bacteriae, like C. pneumoniae, in atherogenesis and the occurrence of adverse events. The present observation suggests that the role of gram-positive bacteriae and their most recognized ligand, PGN, need to be taken into account when the role of bacterial load in atherogenesis is discussed.

In summary, in atherosclerotic patients systemic IgM antibodies against the proinflammatory antigen PGN are significantly lower compared to control patients with increased cardiovascular risk but without clinically manifest disease, perhaps due to preferential downregulation of the IgM antibody response. This result supports the idea that immune modulation may affect the progression of atherosclerotic disease. Further studies are required to assess which mechanism underlies lower IgM levels, and whether IgM directed against PGN is beneficial for example by reducing its access to plaques.

This is a cross-sectional study. Therefore, this study cannot exclude the possibility that the decrease in IgM antibody levels against PGN is a consequence of the atherosclerotic process itself. To show that the levels of IgM antibody titers against PGN are preceding the atherosclerotic process and thereby contribute to atherosclerosis, prospective studies need to be performed.

\section{Acknowledgements}

This study was supported by the Dutch Heart Foundation (grant 2001B077). The SMART study is supported by a grant of the University Medical Center Utrecht. The authors gratefully thank the Department of Medical Immunology, University Medical Center Utrecht for technical assistance during total immunoglobulin determinations.

\section{References}

[1] Ross RR. Atherosclerosis-an inflammatory disease. New Engl J Med 1999;340:115-26.

[2] Glass CK, Witztum JL. Atherosclerosis: the road ahead. Cell 2001;104:503-16.

[3] Schrijver IA, Melief MJ, Eulderink F, Hazenberg MP, Laman JD. Bacterial peptidoglycan-polysaccharides in sterile human spleen induce proinflammatory cytokine production by human blood cells. J Infect Dis 1999;179:1459-68.

[4] Martinez-Martinez L, Timmerman CP, Fleer A, Verhoef J. Chemiluminescence of human polymorphonuclear leukocytes after stimulation with whole cells and cell-wall components of Staphylococcus epidermis. J Med Microbiol 1993;39:196-203.

[5] Dobrina A, Nardon E, Vecile E, Cinco M, Patriarca P. Leptospira icterohemorrhagiae and leptospire peptidoglycans induce endothelial cell adhesiveness for polymorphonuclear leukocytes. Infect Immun 1995;63:2995-9.

[6] Rasanen L, Lehto M, Jokinen I, Leinikki P. Polyclonal antibody formation of human lymphocytes to bacterial components. Immunology 1986;58:577-81.
[7] Wang JE, Jørgenson PF, Almlöf M, Solberg R, Okkenhaug C, Scholtz $\mathrm{T}$, et al. Peptidoglycan and lipoteichoic acid from Staphylococcus aureus induce tumor necrosis factor alpha, interleukin 6 (IL-6), and IL-10 production in both $\mathrm{T}$ cells and monocytes in a human whole blood model. Infect Immun 2000;68:3965-70.

[8] Yoshimura A, Lien E, Ingalls RR, Tuomanen E, Dziarsky R, Golenbock D. Cutting edge: recognition of gram-positive bacterial cell wall components by the innate immune system occurs via Toll-like receptor 2. J Immunol 1999;163:1-5.

[9] Kaul D. Molecular link between cholesterol, cytokines and atherosclerosis. Mol Cell Biochem 2001;219:65-71.

[10] Laman JD, Schoneveld AH, Moll FL, Van Meurs M, Pasterkamp G. Significance of peptidoglycan, a proinflammatory bacterial antigen in atherosclerotic arteries and its association with vulnerable plaques. Am J Cardiol 2002;90:119-23.

[11] Schrijver IA, de Man Y, Melief MJ, Van Laar JM, Markusse HM, Klasen IS, et al. Reduced systemic IgG levels against peptidoglycan in rheumatoid arthritis (RA) patients. Clin Exp Immunol 2001;123:140-6.

[12] Schrijver IA, Melief MJ, Tak PP, Hazenberg MP, Laman JD. Antigen-presenting cells containing bacterial peptidoglycan in synovial tissues of rheumatoid arthritis patients coexpress costimulatory molecules and cytokines. Arthr Rheum 2000;43:2160-8.

[13] Simons PC, Algra A, van de Laak MF, Grobbee DE, van der Graaf Y. Second Manifestations of ARTerial disease (SMART) study: rationale and design. Eur J Epidemiol 1999;15:773-81.

[14] Hazenberg MP, Pennock-Schroder AM, Wensinck F, Van de Merwe JP. Effect of a soluble bacterial carbohydrate fraction on the viscosity of intestinal contents in healthy subjects and patients with Crohn's disease. Eur J Clin Invest 1989;19:61-4.

[15] Hazenberg MP, de Visser H, Bras MJ, Prins ME, Van de Merwe JP. Serum antibodies to peptidoglycan-polysaccharide complexes from the anaerobic intestinal flora in patients with Crohn's disease. Digestion 1990;47:172-80.

[16] Simons PC, Algra A, Bots ML, Banga JD, Grobbee DE, van der Graaf Y. for the SMART Study Group. Common carotid intima-media thickness and arterial stiffness: indicators of cardiovascular risk in high-risk patients. The SMART Study (Second Manifestations of ARTerial disease). Circulation 1999;100:951-7.

[17] Brandtzaeg P. The role of humoral mucosal immunity in the induction and maintenance of chronic airway infections. Am J Respir Crit Care Med 1995;151:2081-6.

[18] Hulthe J, Wikstrand J, Lidell A, Wendelhag I, Hansson GK, Wiklund O. Antibody titers against oxidized LDL are not elevated in patients with familial hypercholesterolemia. Arterioscler Thromb Vasc Biol 1998;18:1203-11.

[19] Hulthe J, Bokemark L, Fagerberg B. Antibodies to oxidized LDL in relation to intima-media thickness in carotid and femoral arteries in 58-year-old subjectively clinically healthy men. Arterioscler Thromb Vasc Biol 2001;21:101-7.

[20] Wu R, de Faire U, Lemne C, Frostegård J. Autoantibodies to Ox-LDL are decreased in individuals with borderline hypertension. Hypertension 1999;33:53-9.

[21] Ameli S, Hultgårdh-Nilsson A, Regnström J, Calara F, Yano J, Cercek B, et al. Effect of immunization with homologous LDL and oxidized LDL on early atherosclerosis in hypercholesterolemic rabbits. Arterioscler Thromb Vasc Biol 1996;16:1074-9.

[22] Maron R, Sukhova G, Faria AM, Hoffmann E, Mach F, Libby $P$, et al. Mucosal administration of heat shock protein-65 decreases atherosclerosis and inflammation in aortic arch of low-density lipoprotein receptor-deficient mice. Circulation 2002;106:1708-15.

[23] Caligiuri G, Nicoletti A, Poirier B, Hansson GK. Protective immunity against atherosclerosis carried by B cells of hypercholesterolemic mice. J Clin Invest 2002;109:745-53.

[24] Shaw PX, Hörkkö S, Chang MK, Curtiss LK, Palinski W, Silverman GJ, et al. Natural antibodies with the T15 idiotype may act in 
atherosclerosis, apoptotic clearance, and protective immunity. J Clin Invest 2000;105:1731-40.

[25] Virella G, Lopes-Virella MF. Humoral immunity and atherosclerosis. Natl Med 2003;9:243-4.

[26] Schumacher A, Seljeflot I, Lerkerod AB, Sommervoll L, Otterstad JE, Arnesen H. Positive Chlamydia pneumoniae serology is associated with elevated levels of tumor necrosis factor alpha in patients with coronary heart disease. Atherosclerosis 2002;164:153-60.

[27] Schumacher A, Seljeflot I, Lerkerod AB, Sommervoll L, Otterstad JE, Arnesen H. Does infection with Chlamydia pneumoniae and/or Helicobacter pylori increase the expression of endothelial cell adhesion molecules in humans? Clin Microbiol Infect 2002;8: 654-61.
[28] Siscovick DS, Schwartz SM, Corey L, Grayston JT, Ashley R, Wang $\mathrm{S}-\mathrm{P}$, et al. Chlamydia pneumoniae, Herpes simplex virus type 1, and cytomegalovirus and incident myocardial infarction and coronary heart disease death in older adults. The Cardiovascular Health Study. Circulation 2000;102:2335-40.

[29] Kowalski M, Rees W, Konturek PC, Grove R, Scheffold T, Meixner $\mathrm{H}$, et al. Detection of Helicobacter pylori specific DNA in human atheromatous coronary arteries and its association to prior myocardial infarction and unstable angina. Dig Liver Dis 2002;34:398-402.

[30] De Backer J, Mak R, De Bacquer D, Van Renterghem L, Verbaekel E, Kornitzer M, et al. Parameters of inflammation and infection in a community based case-control study of coronary heart disease. Atherosclerosis 2002;160:457-63. 